\title{
Blood Cultures for Community-Acquired Pneumonia: Are They Worthy of Two Quality Measures? A Systematic Review
}

\author{
Nima Afshar, MD ${ }^{1,2}$ \\ Jeffrey Tabas, MD ${ }^{1}$ \\ Kia Afshar, $\mathrm{MD}^{3,4}$ \\ Robert Silbergleit, $\mathrm{MD}^{2}$ \\ ${ }^{1}$ Department of Medicine, University of Califor- \\ nia, San Francisco, San Francisco, California. \\ 2 Department of Emergency Medicine, University \\ of Michigan, Ann Arbor, Michigan. \\ ${ }^{3}$ Department of Emergency Medicine, University \\ of California, San Fransisco, San Fransisco, Cali- \\ fornia. \\ ${ }^{4}$ Department of Medicine, Johns Hopkins Hospi- \\ tal, Baltimore, Maryland.
}

BACKGROUND: Obtaining blood cultures (BCs) for patients hospitalized with community-acquired-pneumonia (CAP) has been recommended by experts and used as a measure of quality of care. However, BCs are infrequently positive in these patients and their effect on clinical management has been questioned.

PURPOSE: We performed a systematic review of the literature to determine the impact of BCs on clinical management in CAP requiring hospitalization and thus its appropriateness as a quality measure.

DATA SOURCES: We searched MEDLINE, MEDLINE In-Process, and the Cochrane databases for English-language studies that reported the effect of BCs on management of adults hospitalized with CAP. We also searched the reference lists of included studies and background articles and asked experts to review our list for completeness.

STUDY SELECTION: Studies were chosen if they included adults admitted to the hospital with CAP, BCs were obtained at admission, and BC-directed management changes were reported.

DATA EXTRACTION: We abstracted study design, BC positivity, and frequency of BC-directed management changes.

DATA SYNTHESIS: Fifteen studies, all with observational cohort design, were identified and reviewed. Two included only patients with BCs positive for pneumococcus, yielding 13 studies for the primary analysis. BCs were true-positive in $0 \%$ to $14 \%$ of cases. They led to antibiotic narrowing in $0 \%$ to $3 \%$ of patients and to antibiotic broadening ultimately associated with a resistant organism in $0 \%$ to $1 \%$ of patients.

CONCLUSIONS: BCs have very limited utility in immunocompetent patients hospitalized with CAP. Pneumonia quality measures that include BCs should be reassessed. Journal of Hospital Medicine 2009;4:112-123. ๑ 2009 Society of Hospital Medicine.

KEYWORDS: pneumonia, blood cultures, antibiotics, quality measures.

D lood cultures (BCs) have long been a mainstay of the diagnostic evaluation of patients hospitalized with communityacquired pneumonia (CAP). They have been strongly recommended by professional societies ${ }^{1-3}$ and are often expected by admitting physicians. A large retrospective study of Medicare patients with pneumonia found that obtaining BCs is associated with lower mortality. ${ }^{4}$ In 2002, when the National Hospital Quality Measures (NHQM) were introduced, BCs were included as a quality measure for pneumonia. ${ }^{5,6}$
There was no external funding source for this systematic review. 
However, there is uncertainty about the actual utility of BCs in CAP. In large studies they are true-positive in only 7 to $11 \%$ of cases and falsepositive in $\sim 5 \%,{ }^{2,7}$ and whether they affect clinical management has been strongly questioned. ${ }^{8-10}$ Their impact may be limited by slow results, low frequency of bacterial resistance to the empiric antibiotic regimen, and reluctance of physicians to narrow antibiotic coverage. ${ }^{9,11}$ Recent updates to professional society guidelines no longer recommend BCs in all admitted CAP patients. ${ }^{12}$

To evaluate the clinical utility of BCs and the appropriateness of pnemonia quality measures based on BCs, we performed a systematic review of the literature to determine the effect of BCs on the management of adults with CAP requiring hospitalization.

\section{PATIENTS AND METHODS \\ Data Sources and Searches}

We searched the English-language literature via MEDLINE (1966 through September 2007), MEDLINE-In Process, Cochrane Central Register of Controlled Trials, Cochrane Database of Systematic Reviews, Database of Abstracts of Reviews of Effects, and ACP Journal Club. Within each of these databases we used keywords and exploded Medical Subject Headings (MeSH) to produce the following search strategy: blood culture(s) (keyword), bacteriological techniques (MeSH), blood [microbiology] (MeSH), bacteremia [microbiology or drug therapy] (MeSH), or diagnostic tests, routine (MeSH) combined with pneumonia (keyword), pneumonia $(\mathrm{MeSH})$, lower respiratory tract infection(s) (keyword), or community-acquired infections (MeSH). To maximize capture of $\mathrm{BC}$ or bacteremia studies with subgroups of CAP patients we added the following search strategy: explode microbiological techniques [utilization] (MeSH), explode blood specimen collection [utilization] (MeSH), or focus bacteremia [drug therapy] (MeSH). We reviewed the reference lists of all included studies as well as those of important background articles. Finally, we asked experts to evaluate the completeness of our list.

\section{Study Selection}

We included studies in which: (1) subjects were adults hospitalized with CAP; (2) BCs were obtained at or near hospital admission; and (3) the effects of BCs on management (change in an- tibiotic therapy or other effects such as duration of parenteral therapy, length of hospitalization, or level of care) were reported. The first 2 requirements could be satisfied by a subgroup.

From retrieved citations, relevant abstracts were reviewed, and studies with any potential to meet inclusion criteria were chosen for full-text review. Two authors (N.A., R.S.) independently analyzed each full-text article to determine inclusion for data analysis. A third author (J.T.) analyzed all included and narrowly excluded articles to confirm the final list of included studies. Disagreements were resolved by discussion.

\section{Data Extraction}

For the included studies, 2 authors (N.A., K.A.) independently abstracted the following data using a standardized collection instrument: study design and setting, inclusion and exclusion criteria, number of hospitalized CAP patients in whom BCs were obtained, empiric antibiotic regimens, number of true-positive and false-positive BCs, bacteria isolated in true-positive $\mathrm{BCs}, \mathrm{BC}$-directed antibiotic narrowing, BC-directed antibiotic broadening ultimately associated with a resistant organism, and any other management effects reported. "Narrowing" refers to coverage of fewer organisms, while "broadening" refers to coverage of a larger or different spectrum of organisms.

If a study included patients not meeting our selection criteria, our analysis was limited to the subset of patients meeting criteria. We also analyzed each study to determine whether a subgroup of severely ill patients was reported separately and whether such a group benefited from BCs. The 2 authors independently repeated all data abstraction to confirm accuracy. We attempted to contact authors for clarification when needed.

\section{Data Synthesis}

Data were synthesized by compilation of characteristic summary tables. In the primary analysis, the proportion of positive $\mathrm{BCs}$ (both true and false) and the frequency of BC-directed changes in antimicrobial therapy (narrowing, or broadening ultimately associated with a resistant organism) were determined and reported for each study and then described as an aggregate range. This compilation required studies to provide a particular denominator-the number of patients in whom BCs 
were performed. If a study did not do so, it was described separately in the secondary analysis, where we also assessed the cost of BCs as well as the impact of BCs in critically ill patients and on outcomes other than antibiotic change. Heterogeneity of subject inclusion and exclusion criteria and empiric antibiotic use were summarized qualitatively. Two authors (N.A., R.S.) assessed each study's quality.

\section{DATA SYNTHESIS}

\section{Search Results}

Our electronic database search yielded 3236 citations. From this list and the supplementary search of references, we reviewed 607 abstracts; of these, we selected 73 articles for full-text review, and 15 were included in the final analysis (Figure 1). One study was narrowly excluded because it largely included CAP patients that had already been admitted to the hospital and failed an empiric antibiotic trial before BCs were obtained. ${ }^{13}$

\section{Study Characteristics}

Fifteen studies with a total of 3898 patients evaluated $\mathrm{BC}$-directed management changes in adults admitted with CAP ${ }^{11,14-27}$ However, 2 of these, involving only patients with bacteremic pneumococcal CAP, by design could not report the number of patients that had BCs done; thus they were not included in the primary analysis. ${ }^{16,25}$

The 13 studies amenable to the primary analysis (Table 1) all had an observational cohort design; 6 were prospective ${ }^{11,18,20,24,26,27}$ and 7 were retrospective. ${ }^{14,15,17,19,21-23}$ Sample size varied from 52 to 760 patients. Settings included university and community hospitals in the U.S. and 4 other countries, with patient enrollment spanning the years 1988-2003 (publication dates 1991-2007).

Included patients were usually required to have clinical features of pneumonia and a confirmatory chest $\mathrm{x}$-ray. Treating physicians were required to obtain BCs (either by study or hospital protocol) in only 3 studies ${ }^{14,22,24}$ and in a subgroup of another study; ${ }^{11}$ otherwise the performance of BCs was left to physician discretion.

Nine studies excluded patients who were immunocompromised, ${ }^{11,15,17,18,22-24,26,27}$ a label that was often incompletely defined. Otherwise, exclusion criteria were variable. Notably, only 3 studies excluded patients admitted to the inten-

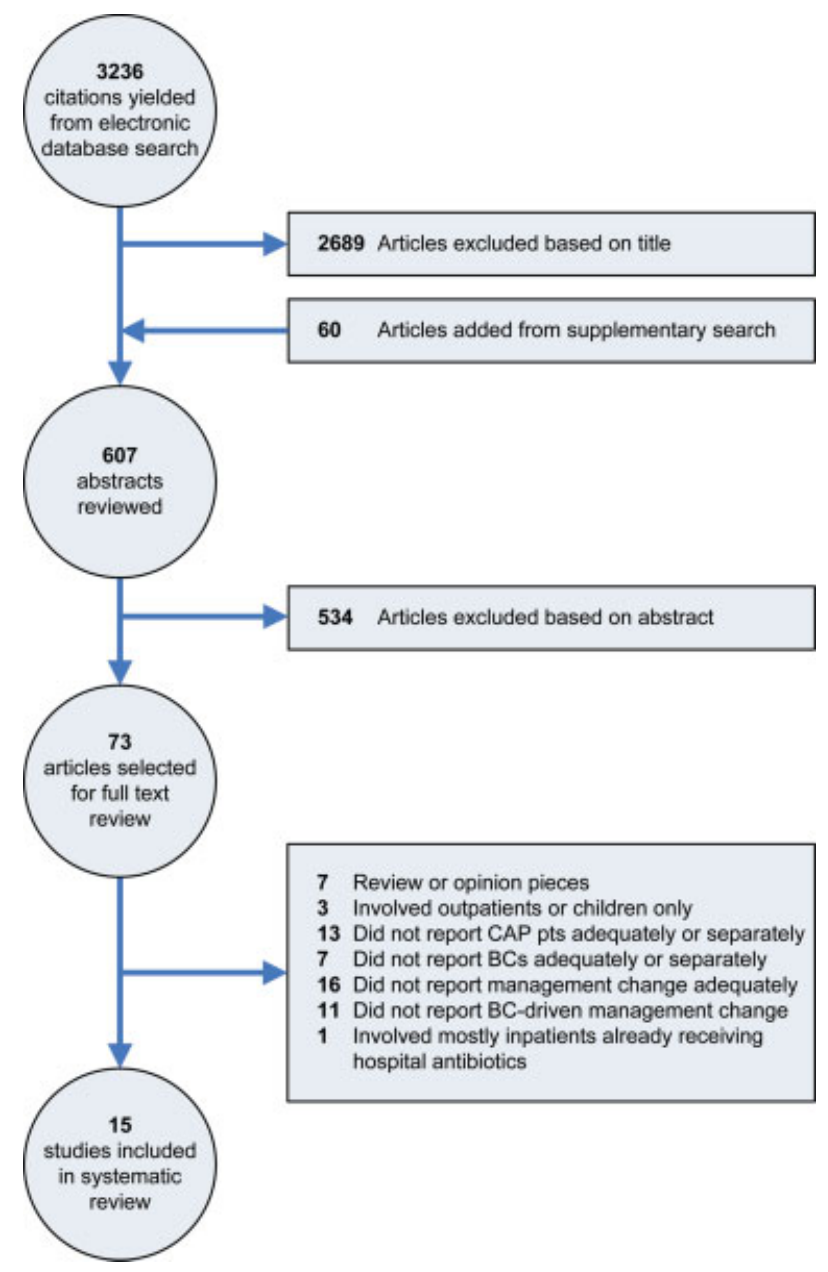

FIGURE 1. Study selection process.

sive care unit (ICU), 11,18,24 while 6 excluded patients with cancer ${ }^{15,17,22-24,27}$ and 6 excluded either nursing home residents ${ }^{15,17,22,26}$ or the elderly (de facto exclusion of most nursing home residents). ${ }^{24,27}$

Empiric antibiotic regimens, where reported, were predominantly cephalosporin plus macrolide combinations in 4 studies, ${ }^{17,22-24}$ fluoroquinolones in 3 studies, ${ }^{11,14,26}$ and penicillin or 1 of its derivatives in 1 study. ${ }^{27}$

Concerning the 2 studies not included in the primary analysis, the one by Waterer et $\mathrm{al}^{25}$ was a retrospective review of all cases of pneumococcal bacteremia $(\mathrm{n}=74)$ associated with an admission diagnosis of CAP $(\mathrm{N}=1805)$ in a US urban hospital over a 3-year period. The one by Chang et al. ${ }^{16}$ was a retrospective case-control study of 288 ran- 


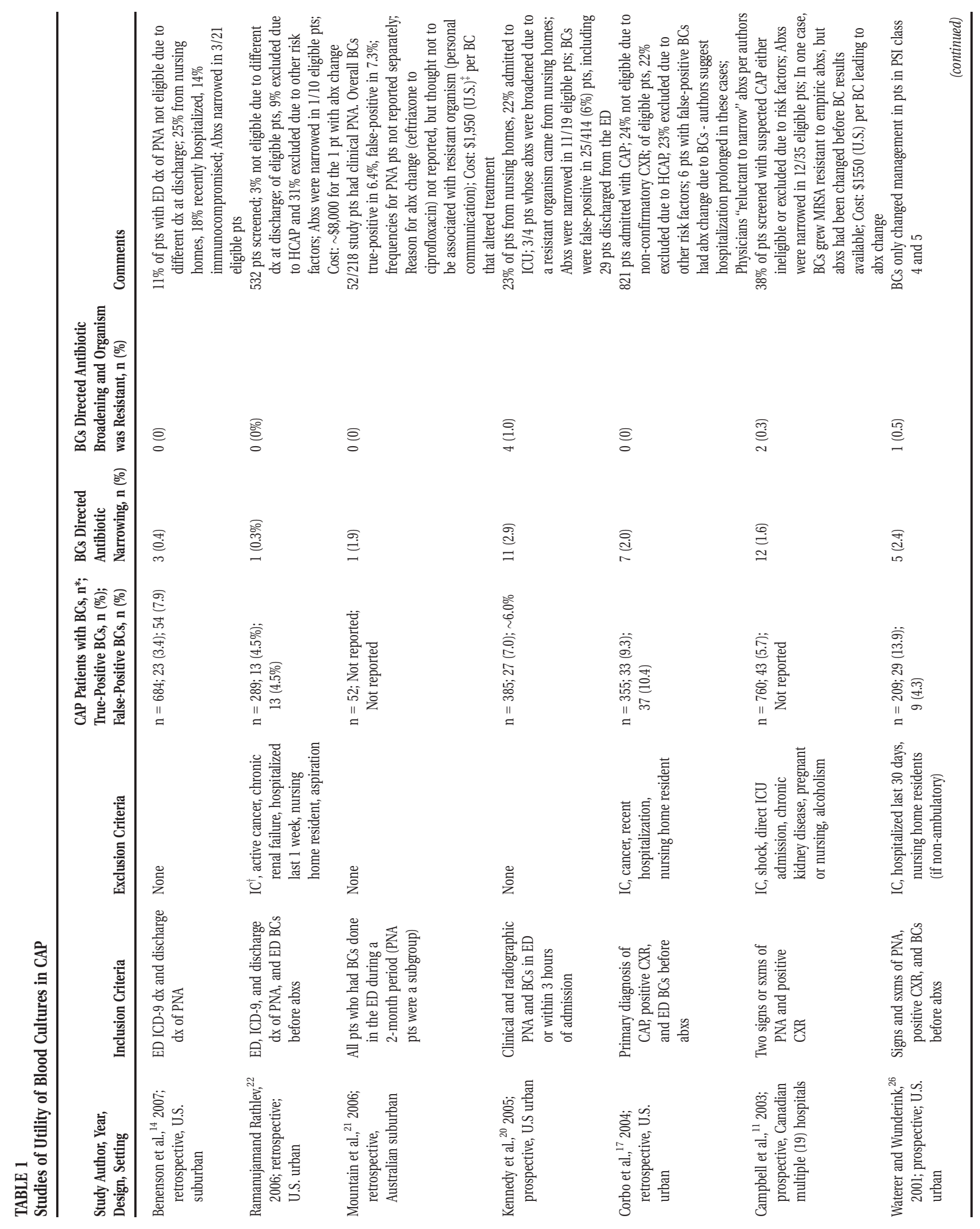




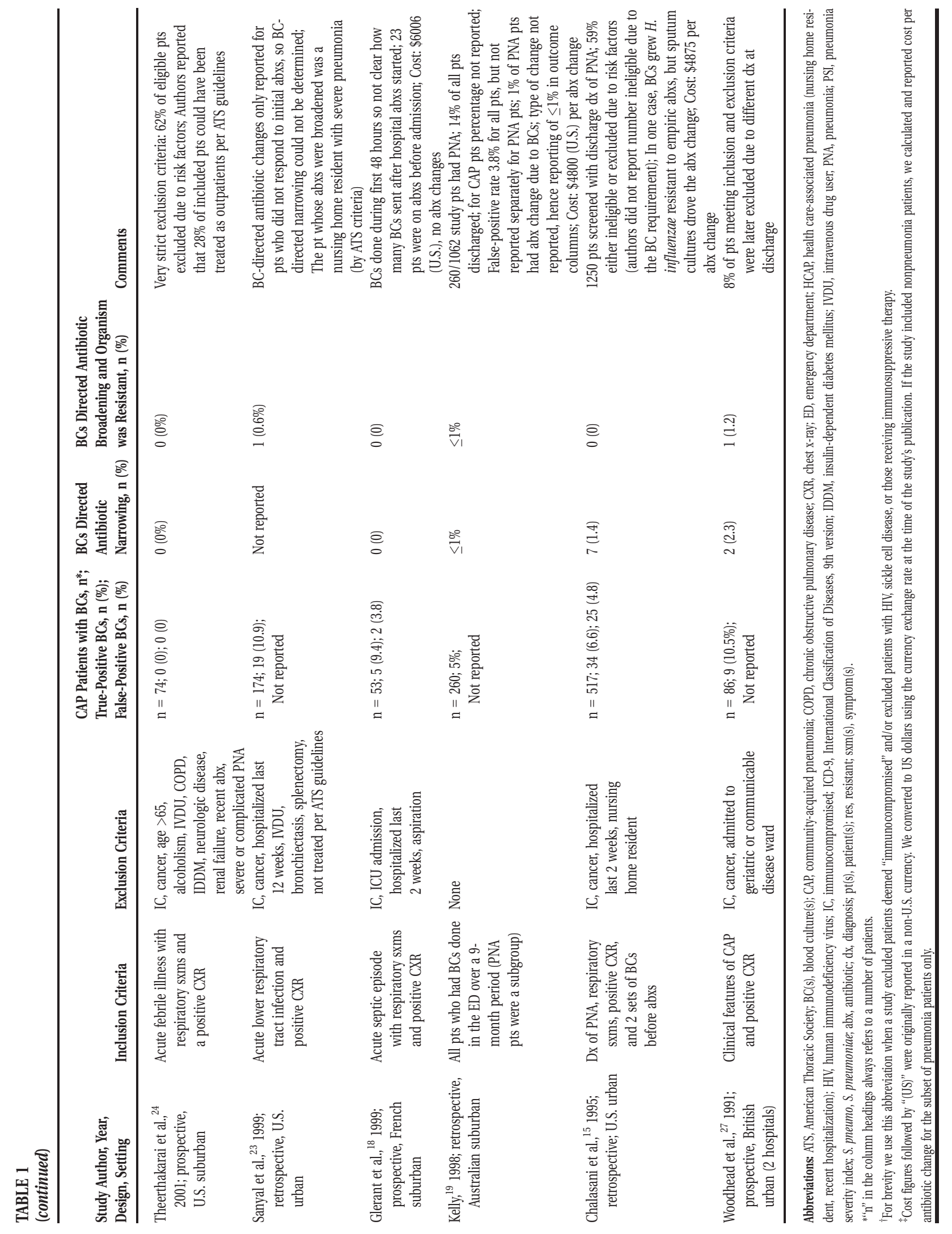


domly-selected, immunocompetent Medicare patients with bacteremic pneumococcal CAP who survived to discharge. They were matched 1:1 with blood and sputum culture-negative controls to study the rate of fluoroquinolone use at discharge in the 2 groups.

\section{Study Findings \\ Primary Analysis}

As shown in Table 1, BCs were positive for a true pathogen in $0 \%$ to $14 \%$ of cases. Details of microbiology and empiric antibiotic selection are reported in Table 2. S. pneumoniae was by far the most common pathogen: of the 9 studies that had positive BCs and reported the organisms, S. pneumoniae represented $50 \%$ to $91 \%$ of the pathogens, with penicillin-resistance found in $0 \%$ to $20 \% .{ }^{11,14,15,17,18,20,22,23,26}$ S. aureus was next most common, occurring in 6 studies and growing in $3 \%$ to $23 \%$ of positive $\mathrm{BCs} ;{ }^{11,14,17,20,23,26}$ its sensitivity to methicillin was reported in 3 studies, with methicillin-resistant $S$. aureus (MRSA) representing $0 / 3,3 / 7$, and $1 / 1$ of cases. ${ }^{14,20,23}$ E. coli represented $3 \%$ to $11 \%$ of pathogens in 6 studies, ${ }^{11,14,15,20,23,26}$ while $H$. influenzae represented $2 \%$ to $15 \%$ of pathogens in 7 studies. ${ }^{11,14,15,18,22,23,26}$

In the 8 studies that reported false-positive BCs, the false-positive rate was $0 \%$ to $10 \%,{ }^{14,15,17,18,20,22,24,26}$ with 5 studies finding comparable false-positive and true-positive BC rates ${ }^{15,17,20,22,24}$ and 1 study finding a substantially higher frequency of false-positive than true-positive BCs (Table 1$).{ }^{14}$

BCs led to narrowing of antibiotic coverage in $0 \%$ to $3 \%$ of cases (Table 1). Four studies reported that physicians narrowed antibiotics when BCs indicated that it was possible to do so, but only in $10 \%, 14 \%, 34 \%$, and $58 \%$ of eligible cases. ${ }^{11,14,20,22}$

BCs led to antibiotic broadening ultimately associated with a resistant organism in $0 \%$ to $1 \%$ of cases (Table 1). The pathogens were MRSA (3), methicillin-sensitive S. aureus (2), E. coli (2), S. pneumoniae (1), and Group D Streptococcus (1). Details about these patients' medical histories and demographics were absent or sparse in all but 1 study. ${ }^{20}$ For several of the above cases it was not explicitly stated that BCs directed the antibiotic changes, though it was usually implied; thus we assumed causation.

\section{Secondary Analyses}

In the pneumococcal bacteremia study by Waterer et al., ${ }^{25}$ BCs altered management in 31 of the 74 cases of pneumococcemia, but in only 2 patients was this associated with antibiotic resistance. Most of the other 29 cases involved narrowing of antibiotics, though switching to penicillin or dropping atypical coverage occurred in only $22 \%$ and $37 \%$ of eligible patients, respectively. In the study by Chang et al., ${ }^{16}$ there was no significant difference in fluoroquinolone use at discharge between the pneumococcemic and culture-negative groups (the primary endpoint), though there was significantly higher $\beta$-lactam use and lower macrolide use in the pneumococcemic patients at discharge. From the data provided it was not possible to determine how often antibiotic broadening occurred.

Only 2 of the 15 studies stratified management effects based on severity of illness, and neither specified the proportion of severely ill patients admitted to the ICU. Waterer and Wunderink ${ }^{26}$ prospectively hypothesized that sicker patients were more likely to benefit from BCs. They found that the 30 patients in pneumonia severity index class 5 were most likely to have a BC-driven antibiotic change, though in at most 1 of these patients was associated with a resistant organism. Sanyal et al. ${ }^{23}$ stratified patients by severity based on expert guidelines. They found that 19 of 174 patients had severe CAP that did not respond to the initial antibiotic regimen, with 1 having a BC-driven antibiotic change; this was due to resistance.

Only 1 study reported an outcome other than antibiotic change, which in this case was duration of parenteral therapy. In the study, 5 of 43 patients with true-positive BCs remained on intravenous antibiotics for the full course of treatment probably due to bacteremia alone. ${ }^{11}$

The direct cost of BCs per BC-directed antibiotic change (or total cost of BCs if there was no antibiotic change) was reported in 6 studies and, not adjusted for inflation, ranged from $\$ 1550$ to $\$ 8000$ (U.S.). ${ }^{11,15,18,19,21,22}$

\section{Quality of the Studies}

A detailed listing of the strengths and weaknesses of each study is provided in the Appendix. Briefly, all 15 studies included in this review were observational. Most did not prospectively require BCs 


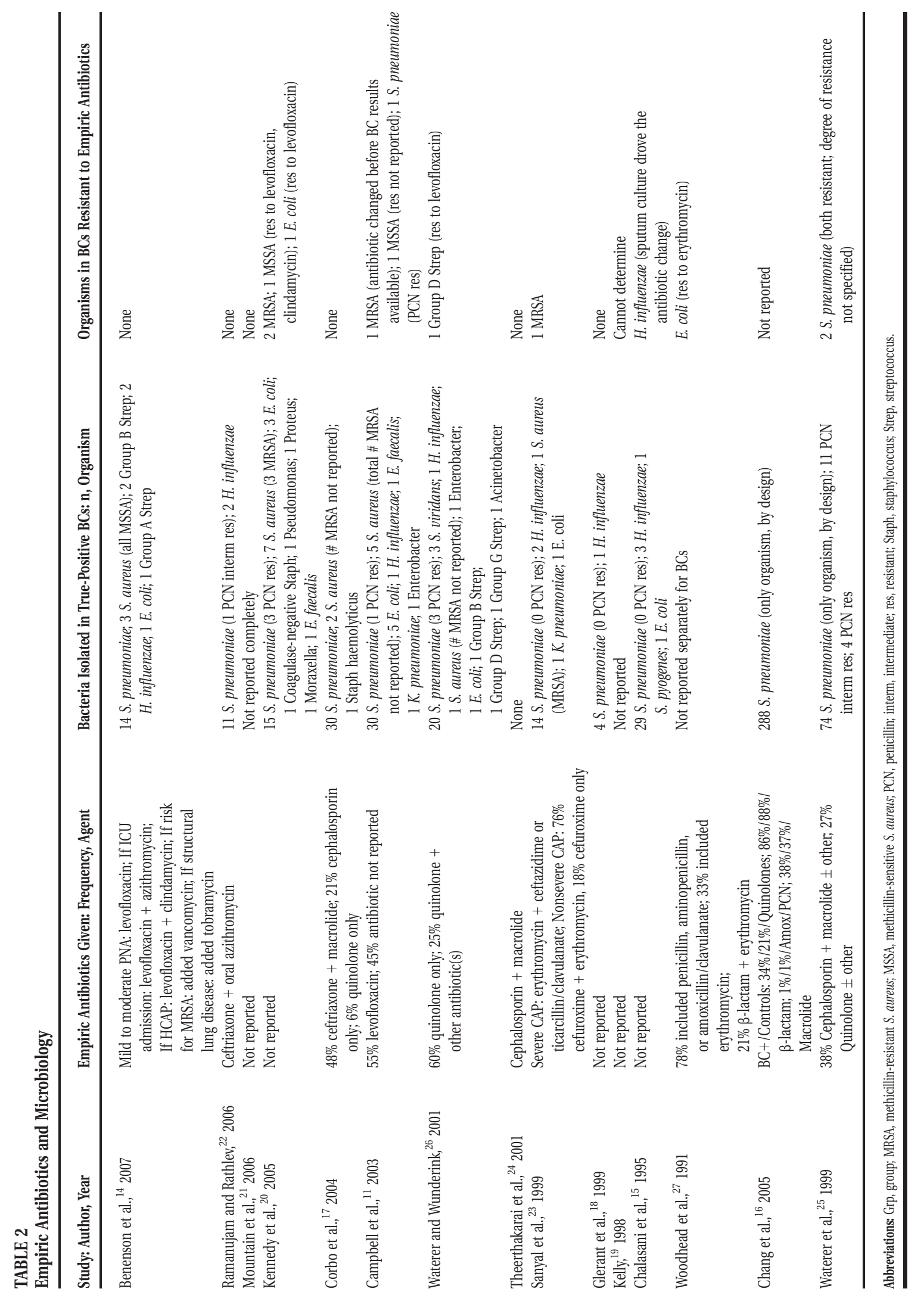


in all patients admitted with CAP. This could have biased the results in favor of BC utility as physicians presumably order BCs in patients with a higher probability of bacteremia. Conversely, several studies did not explicitly require two sets of $\mathrm{BCs}$ or that BCs be done prior to antibiotics, so they may not have revealed the maximum utility of BCs. The 2 studies limited to pneumococcal bacteremia and described in the secondary analysis were inherently biased against BC utility, as pneumococcus is more likely to be antibiotic-sensitive than other CAP pathogens.

Eligibility was based only on an emergency department (ED)/admission diagnosis of CAP, a criteria that approximates real world practice, in 3 studies. ${ }^{19,21,25}$ The other studies required either a confirmatory radiograph or a hospital discharge diagnosis of pneumonia. Consequent ED/admission misdiagnosis rates were $3 \%, 8 \%, 11 \%, 24 \%$ in the 4 studies that reported them; ${ }^{14,17,22,27}$ the final diagnoses, when reported, were nearly all noninfections or proximal respiratory tract infections. ${ }^{22,27}$

Five studies included all eligible patients. ${ }^{14,19-21,25}$ However, 3 studies excluded $23 \%, 31 \%$, and $62 \%$ of eligible patients based on risk factors for bacteremia or resistant pathogens, ${ }^{17,22,24}$ and the rest did not report the number excluded.

\section{DISCUSSION \\ Summary of Findings}

Our systematic review of the literature finds that BCs rarely alter empiric antibiotic therapy in adults hospitalized with community-acquired pneumonia. Even when there is a change in treatment it usually is not of the type most likely to impact patient outcome, which is antibiotic broadening ultimately associated with a resistant organism. In the 13 studies that could quantify this effect, it occurred in only $0 \%$ to $1 \%$ of cases in which BCs were obtained. Antibiotic narrowing occurred in $0 \%$ to $3 \%$ of cases, with physicians often choosing not to narrow antibiotics when $\mathrm{BC}$ results suggested that they could do so.

\section{Limits on BC Utility \\ $\beta$-Lactam-Resistant Pneumococcus}

In the studies reviewed here $50 \%-90 \%$ of positive BCs grew pneumococcus, consistent with the $60 \%$ to $67 \%$ rate reported elsewhere. ${ }^{2,28,29}$ Pneumococci that invade the bloodstream have disproportio- nately low rates of $\beta$-lactam resistance, ${ }^{30,31}$ inherently limiting the utility of BCs for detecting inadequate empiric antibiotic therapy. Though pneumococcal resistance to $\beta$-lactams has risen over the last 2 decades, third-generation cephalosporins, preferred agents for CAP, are still extremely effective. Even when the organism is by historical standards moderately resistant to them, these cephalosporins at standard doses maintain bactericidal efficacy in the lung, ${ }^{32,33}$ and their use in the setting of such resistance is not associated with higher mortality. ${ }^{34-37}$ By newer laboratory standards $97 \%$ and $96 \%$ of S. pneumoniae isolates in mid-2003 were sensitive to ceftriaxone and cefotaxime, respectively. ${ }^{38}$ Thus a major potential benefit of BCs-detecting cephalosporin-resistant pneumococcus-remains a rare occurrence.

\section{Polymicrobial Infection}

If positive $\mathrm{BCs}$ in CAP mostly reveal antibioticsensitive pathogens, one may infer that at least they lead to narrowing of therapy. However, the studies reviewed here reveal that this usually does not happen.

One explanation for this reluctance to narrow antibiotics is that CAP is often a polymicrobial disease. When rigorous serologic testing is done, multiple pathogens are found in up to $40 \%$ of cases. ${ }^{39}$ The occult copathogen is frequently an intracellular one and thus cannot be detected by BCs. Though the evidence for empirically treating these atypical organisms is mixed, ${ }^{40,41}$ expert guidelines recommend doing so, ${ }^{12}$ and guidelineconcordant antibiotic therapy in CAP is associated with lower mortality. ${ }^{42}$ Even in bacteremic pneumococcal CAP, monotherapy is associated with higher mortality. ${ }^{43-46}$ Thus, stopping antibiotic coverage of atypical pathogens in response to BCs alone might not always be appropriate.

\section{Prognosis}

Another rationale given for ordering BCs is that bacteremic pneumonia is a morbid disease so positive BCs may demand prolonged parenteral therapy or extended hospitalization. Although mortality for bacteremic pneumococcal pneumonia (the predominantly studied variety of bacteremic pneumonia) has historically been high at $\sim 20 \%,{ }^{47,48}$ studies that have examined pneumococcal bacteremia as an independent risk factor for death in CAP have yielded mixed results. ${ }^{2}$ 
Moreover, it appears that patients with bacteremic pneumococcal pneumonia who reach clinical stability may be safely switched to oral antibiotics. ${ }^{49}$

It is not clear that positive BCs in pneumonia (at least in the case of S. pneumoniae) should alter the duration of parenteral therapy or hospitalization, though whether or not such effects occur in clinical practice was largely unaddressed by the studies reviewed here.

\section{Epidemiology}

One theoretical benefit of BCs is their epidemiologic value. When true-positive in pneumonia, perhaps more than any other test they identify with great specificity at least 1 of the causative agents. Unfortunately, as discussed above, BCs alone provide an incomplete and skewed picture of the microbiology of CAP. They underestimate atypical organisms, overestimate pneumococcus, and, because bacteremic pneumococcus is more likely to be antibiotic-susceptible, they underestimate antibiotic resistance. ${ }^{11}$ Tracking pathogens in bacteremic pneumonia may be useful nonetheless, but perhaps a more accurate method for determining etiologic trends is periodic comprehensive microbiological investigation, including BCs, sputum/bronchial cultures, and serology.

\section{Costs}

In the studies reviewed here, based on reported costs of $\$ 15$ to $\$ 65$ per set of BCs or per patient, BCs cost $\$ 1550$ to $\$ 8000$ (U.S.) per BC-directed antibiotic change. Considering that very few of these antibiotic changes involved broadening associated with a resistant organism, the cost/ benefit ratio was quite high. Today BCs may be even more expensive, as U.S. hospitals now often charge over $\$ 150$ per set of BCs. ${ }^{50,51}$

The cost of false-positive BCs must also be taken into account. The false-positive rate in the studies reviewed here was $0 \%$ to $10 \%$, similar to that reported elsewhere. ${ }^{7} \quad$ False-positive BCs increase hospital length of stay by 3 to 5 days and hospital charges by $\$ 4400$ to $\$ 8800 .^{51,52}$

\section{Limitations of the Review}

Our search strategy was designed to be sensitive and included backup methods such as searching article references and querying experts. Nevertheless, we may have missed studies, especially if there were small eligible subgroups or if determin- ing management effects was not a primary purpose. We chose not to measure instances of antibiotic broadening that were not associated with a resistant organism, though in unusual cases (eg, Pseudomonas bacteremia) this effect of BCs may be useful.

The methodologies of the included studies were adequate to measure the key outcomes with reasonable validity. Biases were evident, though they occurred both for and against BC utility.

Eligibility varied across studies, and most investigations excluded immunocompromised or other high-risk patient groups, which could have biased results against BC utility. However, results of these studies were consistent with those that included all patients with CAP, suggesting the degree of bias was probably small. Still, given this concern, it would be prudent not to generalize the findings of this review to immunocompromised patients. Moreover, although the critically ill and those who today would be classified as having healthcare-associated pneumonia (HCAP)—nursing home residents, the recently hospitalized, and hemodialysis patients-were included in most studies, their numbers were small, and these groups were not analyzed separately; thus, the results might not be generalizable to these populations either. Finally, the reported studies, which enrolled patients through 2003, do not reflect more recent increases in the prevalence of resistant pathogens, such as MRSA, in the community.

\section{BCs as a Quality Measure}

The adoption of BCs as a quality measure was largely predicated on the widely-cited study by Meehan et al., ${ }^{4}$ which showed an association between BC obtainment and reduced mortality. This study, which associated processes of care with hard outcomes such as mortality, was limited by uncontrolled confounders, including variation in hospital quality. ${ }^{53} \mathrm{~A}$ more recent study of pneumonia processes of care found no association between BC collection and mortality. ${ }^{54}$ Another study often cited to support BC use, by Arbo and Snydman, ${ }^{55}$ showed that positive BCs were associated with changes in antibiotic therapy, but it included very few pneumonia patients and did not describe results for them separately.

The inclusion of BC acquisition in 2 quality measures in the NHQM guidelines for pneumonia impacts the clinical practice of hospitals and phy- 
sicians, which may be rated and reimbursed differentially based on their compliance with such measures. One of the quality measures requires BCs in patients admitted to the ICU. The other requires that $\mathrm{ED}$ BCs for pneumonia, if obtained, be drawn before antibiotics are given. ${ }^{6}$

The studies we reviewed are not specific to these quality measures, but are relevant to them. With regard to the first measure, all but 3 studies included patients admitted to the ICU and found BCs to be of minimal benefit overall. Our subgroup analysis of severely ill patients was unrevealing. The ICU measure is tentative in its validity, but it is not unreasonable given that these patients have a life-threatening infection and may be at risk for bacteremia with resistant pathogens. ${ }^{12}$

The second measure, though perhaps simply seeking to maximize the potential for BCs to turn positive, depends for its validity on BCs being useful in a large proportion of patients with CAP. Though we cannot exclude the possibility that BCs benefit certain subsets of patients, such as those who are immunocompromised or have HCAP, our findings do not support obtaining BCs in all or even most adults hospitalized with CAP. This conclusion is reflected in the 2007 Infectious Diseases Society of America/American Thoracic Society management guidelines for CAP, which state than BCs are optional except for patients with severe pneumonia, some immunocompromised states, and particular radiographic abnormalities. ${ }^{12}$

With such data and guidelines in mind, a physician seeking to minimize treatment delays in a patient with pneumonia may give antibiotics early in the ED course (the basis of another quality measure) without obtaining BCs. If she later determines that the patient is particularly high-risk for bacteremia or a resistant pathogen, should she be discouraged from ordering BCs? Experts specifically state that BCs, even after antibiotics, are warranted for such a patient. ${ }^{12}$

With the scope of medical practice captured in quality measures being so narrow, having 2 measures based on a test with such limited benefit is itself questionable.

Address for correspondence and reprint requests: Nima Afshar, MD, Box 0131, 533 Parnassus Ave, UC U-129, University of California, San Francisco, San Francisco, CA 94143-0131; Telephone: (415) 476-4824; Fax: (415) 4764818; E-mail: niafshar@medicine.ucsf.edu

Received 3 July 2007; revision received 23 January 2008; accepted 12 February 2008.

\section{REFERENCES}

1. Bartlett JG, Dowell SF, Mandell LA, File TM Jr, Musher DM, Fine MJ. Practice guidelines for the management of community-acquired pneumonia in adults. Infectious diseases society of America. Clin Infect Dis. 2000;31:347382.

2. Mandell LA, Marrie TJ, Grossman RF, Chow AW, Hyland RH. Canadian guidelines for the initial management of community-acquired pneumonia: an evidence-based update by the Canadian infectious diseases society and the Canadian thoracic society. The Canadian communityacquired pneumonia working group. Clin Infect Dis. 2000; 31:383-421.

3. Niederman MS, Mandell LA, Anzueto A, et al. Guidelines for the management of adults with community-acquired pneumonia. Diagnosis, assessment of severity, antimicrobial therapy, and prevention. Am J Respir Crit Care Med. 2001;163:1730-1754.

4. Meehan TP, Fine MJ, Krumholz HM, et al. Quality of care, process, and outcomes in elderly patients with pneumonia. JAMA. 1997;278:2080-2084.

5. Hospital quality initiative, overview, centers for Medicare and Medicaid services. Available at: http://www.cms. hhs.gov/HospitalQualityInits. Accessed September 2007.

6. Specifications manual for national hospital quality measures, version 2.3b. Available at: http://www.jointcommission. org/PerformanceMeasurement/PerformanceMeasurement/ Current+NHQM+Manual.htm. Accessed October 2007.

7. Metersky ML, Ma A, Bratzler DW, Houck PM. Predicting bacteremia in patients with community-acquired pneumonia. Am J Respir Crit Care Med. 2004;169:342-347.

8. Walls RM, Resnick J. The joint commission on accreditation of healthcare organizations and center for Medicare and Medicaid services community-acquired pneumonia initiative: what went wrong? Ann Emerg Med. 2005;46: 409-411.

9. Luna CM. Blood cultures in community-acquired pneumonia: Are we ready to quit? Chest. 2003;123:977-978.

10. Craven DE. Blood cultures for community-acquired pneumonia: piecing together a mosaic for doing less. Am J Respir Crit Care Med. 2004;169:327-328.

11. Campbell SG, Marrie TJ, Anstey R, Dickinson G, AckroydStolarz S. The contribution of blood cultures to the clinical management of adult patients admitted to the hospital with community-acquired pneumonia: a prospective observational study. Chest. 2003;123:1142-1150.

12. Mandell LA, Wunderink RG, Anzueto A, et al. Infectious Diseases Society of America/American Thoracic Society consensus guidelines on the management of communityacquired pneumonia in adults. Clin Infect Dis. 2007;44 (Suppl 2):S27-S72.

13. Ewig S, Bauer T, Hasper E, Marklein G, Kubini R, Luderitz B. Value of routine microbial investigation in communityacquired pneumonia treated in a tertiary care center. Respiration. 1996;63:164-169.

14. Benenson RS, Kepner AM, Pyle DN, 2nd, Cavanaugh S. Selective use of blood cultures in emergency department pneumonia patients. J Emerg Med. 2007;33:1-8.

15. Chalasani NP, Valdecanas MA, Gopal AK, McGowan JE Jr, Jurado RL. Clinical utility of blood cultures in adult patients with community-acquired pneumonia without defined underlying risks. Chest. 1995;108:932-936. 
16. Chang NN, Murray CK, Houck PM, Bratzler DW, Greenway C, Guglielmo BJ. Blood culture and susceptibility results and allergy history do not influence fluoroquinolone use in the treatment of community-acquired pneumonia. Pharmacotherapy. 2005;25:59-66.

17. Corbo J, Friedman B, Bijur P, Gallagher EJ. Limited usefulness of initial blood cultures in community acquired pneumonia. Emerg Med J. 2004;21:446-448.

18. Glerant JC, Hellmuth D, Schmit JL, Ducroix JP, Jounieaux V. Utility of blood cultures in community-acquired pneumonia requiring hospitalization: Influence of antibiotic treatment before admission. Respir Med. 1999;93:208-212.

19. Kelly AM. Clinical impact of blood cultures taken in the emergency department. J Accid Emerg Med. 1998;15: 254-256.

20. Kennedy M, Bates DW, Wright SB, Ruiz R, Wolfe RE, Shapiro NI. Do emergency department blood cultures change practice in patients with pneumonia? Ann Emerg Med. 2005;46:393-400.

21. Mountain D, Bailey PM, O'Brien D, Jelinek GA. Blood cultures ordered in the adult emergency department are rarely useful. Eur J Emerg Med. 2006;13:76-79.

22. Ramanujam P, Rathlev NK. Blood cultures do not change management in hospitalized patients with communityacquired pneumonia. Acad Emerg Med. 2006;13:740-745.

23. Sanyal S, Smith PR, Saha AC, Gupta S, Berkowitz L, Homel P. Initial microbiologic studies did not affect outcome in adults hospitalized with community-acquired pneumonia. Am J Respir Crit Care Med. 1999;160:346-348.

24. Theerthakarai R, El-Halees W, Ismail M, Solis RA, Khan MA. Nonvalue of the initial microbiological studies in the management of nonsevere community-acquired pneumonia. Chest. 2001;119:181-184.

25. Waterer GW, Jennings SG, Wunderink RG. The impact of blood cultures on antibiotic therapy in pneumococcal pneumonia. Chest. 1999;116:1278-1281.

26. Waterer GW, Wunderink RG. The influence of the severity of community-acquired pneumonia on the usefulness of blood cultures. Respir Med. 2001;95:78-82.

27. Woodhead MA, Arrowsmith J, Chamberlain-Webber R, Wooding S, Williams I. The value of routine microbial investigation in community-acquired pneumonia. Respir Med. 1991;85:313-317.

28. Lim WS, Macfarlane JT, Boswell TC, et al. Study of community acquired pneumonia aetiology (scapa) in adults admitted to hospital: implications for management guidelines. Thorax. 2001;56:296-301.

29. Apisarnthanarak A, Mundy LM. Etiology of communityacquired pneumonia. Clin Chest Med. 2005;26:47-55.

30. Imran MN, Leng PH, Yang S, Kurup A, Eng P. Early predictors of mortality in pneumococcal bacteraemia. Ann Acad Med Singapore. 2005;34:426-431.

31. Winston LG, Perlman JL, Rose DA, Gerberding JL. Penicillin-nonsusceptible Streptococcus pneumoniae at San Francisco general hospital. Clin Infect Dis. 1999;29:580-585.

32. Craig WA. Pharmacokinetic/pharmacodynamic parameters: Rationale for antibacterial dosing of mice and men. Clin Infect Dis. 1998;26:1-10; quiz 11-12.

33. Siegel RE. The significance of serum vs tissue levels of antibiotics in the treatment of penicillin-resistant Streptococcus pneumoniae and community-acquired pneumonia: are we looking in the wrong place? Chest. 1999;116:535-538.
34. Ewig S, Ruiz M, Torres A, et al. Pneumonia acquired in the community through drug-resistant Streptococcus pneumoniae. Am J Respir Crit Care Med. 1999;159:1835-1842.

35. Pallares R, Capdevila O, Linares J, et al. The effect of cephalosporin resistance on mortality in adult patients with nonmeningeal systemic pneumococcal infections. Am J Med. 2002;113:120-126.

36. Pallares R, Linares J, Vadillo M, et al. Resistance to penicillin and cephalosporin and mortality from severe pneumococcal pneumonia in Barcelona, Spain. N Engl J Med. 1995; 333:474-480.

37. Yu VL, Chiou CCC, Feldman C, et al. An international prospective study of pneumococcal bacteremia: correlation with in vitro resistance, antibiotics administered, and clinical outcome. Clin Infect Dis. 2003;37:230-237.

38. Master RN, Draghi DC, Jones ME, Thornsberry C, Sahm DF, Karlowsky JA. Tracking the implementation of NCCLS m100-s12 expanded-spectrum cephalosporin MIC breakpoints for non-meningeal isolates of Streptococcus pneumoniae by clinical laboratories in the united states during 2002 and 2003. Ann Clin Microbiol Antimicrob. 2004;3:1.

39. Lieberman D, Schlaeffer F, Boldur I, et al. Multiple pathogens in adult patients admitted with community-acquired pneumonia: a one year prospective study of 346 consecutive patients. Thorax. 1996;51:179-184.

40. Oosterheert JJ, Bonten MJM, Hak E, Schneider MME, Hoepelman IM. How good is the evidence for the recommended empirical antimicrobial treatment of patients hospitalized because of community-acquired pneumonia? A systematic review. J Antimicrob Chemother. 2003;52: 555-563.

41. Shefet D, Robenshtok E, Paul M, Leibovici L. Empirical atypical coverage for inpatients with community-acquired pneumonia: systematic review of randomized controlled trials. Arch Intern Med. 2005;165:1992-2000.

42. Frei CR, Restrepo MI, Mortensen EM, Burgess DS. Impact of guideline-concordant empiric antibiotic therapy in community-acquired pneumonia. Am J Med. 2006;119:865-871.

43. Baddour LM, Yu VL, Klugman KP, et al. Combination antibiotic therapy lowers mortality among severely ill patients with pneumococcal bacteremia [see comment]. Am J Respir Crit Care Med. 2004;170:440-444.

44. Martinez JA, Horcajada JP, Almela M, et al. Addition of a macrolide to a beta-lactam-based empirical antibiotic regimen is associated with lower in-hospital mortality for patients with bacteremic pneumococcal pneumonia. [see comment]. Clin Infect Dis. 2003;36:389-395.

45. Waterer GW, Somes GW, Wunderink RG. Monotherapy may be suboptimal for severe bacteremic pneumococcal pneumonia. Arch Intern Med. 2001;161:1837-1842.

46. Weiss K, Low DE, Cortes L, et al. Clinical characteristics at initial presentation and impact of dual therapy on the outcome of bacteremic Streptococcus pneumoniae pneumonia in adults. Can Respir J. 2004;11:589-593.

47. Austrian R, Gold J. Pneumococcal bacteremia with special reference to bacteremic pneumococcal pneumonia. Arch Intern Med. 1964;60:759-776.

48. Fine MJ, Smith MA, Carson CA, et al. Prognosis and outcomes of patients with community-acquired pneumonia. A meta-analysis. JAMA. 1996;275:134-141.

49. Ramirez JA, Bordon J. Early switch from intravenous to oral antibiotics in hospitalized patients with bacteremic 
community-acquired Streptococcus pneumoniae pneumonia. Arch Intern Med. 2001;161:848-850.

50. Cleveland Clinic patient price information list. Available at:http://cms.clevelandclinic.org/documents/ CCMain_HB197_2007.pdf. Accessed January 2008.

51. Zwang O, Albert RK. Analysis of strategies to improve cost effectiveness of blood cultures. J Hosp Med. 2006;1:272-276.

52. Bates DW, Goldman L, Lee TH. Contaminant blood cultures and resource utilization. The true consequences of false-positive results. JAMA. 1991;265:365-369.

53. Fine JM, Fine MJ, Galusha D, Petrillo $M$, Meehan TP. Patient and hospital characteristics associated with recommended processes of care for elderly patients hospitalized with pneumonia: results from the Medicare quality indicator system pneumonia module. Arch Intern Med. 2002;162:827-833.

54. Dedier J, Singer DE, Chang Y, Moore M, Atlas SJ. Processes of care, illness severity, and outcomes in the management of community-acquired pneumonia at academic hospitals. Arch Intern Med. 2001;161:20992104.

55. Arbo MD, Snydman DR. Influence of blood culture results on antibiotic choice in the treatment of bacteremia. Arch Intern Med. 1994;154:2641-2645. 